\title{
Identification of Quality Indicators Dynamic System on Basis of Analysis Data "Input-Output"
}

\author{
Nikolay Karabutov \\ Dept. of Problems Control, Moscow state engineering university of radio engineering, electronics and automation, \\ Financial University under the government of the Russian Federation \\ Email: kn22@yandex.ru,nik.karabutov@gmail.com
}

\begin{abstract}
The problem of an estimation quality indicators of linear dynamic system in the conditions of uncertainty is considered. Quality indicators are a point of an equilibrium state and a spectrum of eigenvalues. We offer a method of an estimation a point of an equilibrium state. Method is based on identification of the particular solution system on a class of static models with the dynamic specification on an input. We offered on the basis of the general decision of system procedures and criteria of an estimation equilibrium state. After an estimation of equilibrium state system in work the problem of definition a spectrum eigenvalues of linear dy namic system is considered. We form the time series describing a modification of Lyapunov exponents. For identification of a spectrum eigenvalues we introduce special structures which describe a modification of the Lyapunov exponent. We apply a method of the secant structures and we receive spectrum tentative estimations. The special structure, allowing identifying the largest Lyapunov exponent, is offered. Generalization of the offered methods on linear non-stationary dynamic systems is given.
\end{abstract}

Index Terms - Equilibrium State Point, Spectrum of Eigenvalues, Identification, Structure, Ly apunov Exponents

\section{INTRODUCTION}

At the analysis of quality dynamic systems the great attention is given to stability problems. Method of a phase plane applied to an estimation of a stability system. It allowed to research system behavior about a critical point (equilibrium state). This problem is well researched for systems of the second order in a case when mathematical model [1-6] is known. Performance equation roots on which the inference about critical point type becomes are usually defined. The problem is essentially complicated at the account of nonlinear members of the equation. To decision-making in this case apply the various indirect methods based on an estimation of an index of a critical point, the theorem of Poincare-Bendixs on and others. Except the specified methods can be applied and other approaches. In [7] the problem of identification discrete chaotic maps with critical points is considered. Various models of neural networks are applied to identification of a condition system with their approximation on a specify class of polynomials. It is shown that the combination of these models allows identify ing a critical point the Cusp map.

In [8] application of Poincare index method and DORIC feature for detection of false critical points is described. The approach is realized in system of the ana lysis fingerprints. Critical points on images of fingerprints are studied by many authors [9]. Method of Poincare indexes will be applied to definition of type a critical point at the analysis of images plants the various natures [10]. In [11] by means of Poincare indexes the equilibrium state of dynamic system is analyzed. In [12] the iteration algorithm of definition critical points is offered. Authors understand a critical point as a point which satisfies to the algebraic equations and in which the vector field is not defined.

Other characteristic of dynamic system is the spectrum of eigenvalues. Parametrical methods of identification in a combination to numerical methods are applied to spectrum estimation. Very often for an estimation of stability complicated systems Lyapunov exponents (LE) use. In [13] the algorith $m$ of an evaluation largest LE (LLE) on the basis of the analysis of time series modifications of Dow Jones indexes stock market is offered. LLE is used for a proof of a chaotic modification of Dow Jones inde $\mathrm{x}$ es. In [14] the analysis of methods estimation LE of the distributed systems on the basis of application known methods of an orthogonalization is made. Methods are based on preliminary deriving of trajectories movement system by a solution of the specify equation of a modification dynamics. In [15] it is offered to use LE for testing of condition dynamic systems with one equilibrium state. Authors for definition LE use interpolational methods of the analysis and handling of time series. Application of a graphic mode definition the LLE is given in work [16]. In [17] the problem an estimation of origin chaos in dyna mic system on the basis of a calculation the largest LE is considered. For deriving estimation LLE is analyzed a time series. Other approaches to estimation LE on bases of deriving a solution dynamic system or fulfillment of the analysis experimental data are described in [18-20].

From the reduced analysis we see that stability indicators are applied to an estimation of quality work of dynamic system. For their evaluation or estimation the full a priori information on system is used. In particular, various procedures of handling the time series describing a modification of processes are applied to a calculation of Lyapunov exponents. Researches according to quality of work dynamic system in the conditions of uncertainty or the incomplete a priori information on system on the specified set of indicators were not fulfilled. But there are a class of systems for which the complete mathematical 
description are not known. Application of the specific methods in the absence of the complete the information on mathe matical formu lation of system demanded of preliminary identification of system. Here we have some problems. The problem became complicated, if the information is accessible to observation only in a condition of normal exploitation system. The methods considered above demand the further development.

Considering it, we offer the approached method of an estimation of type an equilibrium state of system in the conditions of uncertainty. It is based on identification of the particular solution of system for the purpose of general solution selection at some in itial condition. For identification of a particular solution of system we used a special class of static models with the dynamic specification on an input. For the first time such approach has been offered in [21]. In work we give generalization of this approach for a case of linear dynamic systems. We reduce procedures and criteria of estimation an equilibrium state on the basis of the system general solution. Then we make the analysis of the received time series and we receive estimations of a spectrum eigenvalues (SE) linear dynamic system. For th is purpose we form the time series describing a modification LE. For identification SE we introduce special structures [21, 22] for describe of modification LE. We apply a method of secants and we receive the approached estimations of a spectrum. The structure for identification LLE is offered.

Work had two parts. The first part contained description of a method identification of an equilibrium state linear dynamic system. The method is based on deriving of estimation a general solution of system. We considered examples of application of a method. Further on the basis of receive general solution of system we considered criteria of an estimation type of an equilibrium state. We gave generalization of a method on a case to an estimation of an equilibrium state linear non-stationary system.

The second part contained description of a method an estimation of a spectrum dynamic system in the conditions of uncertainty. The method gave estimations of LE system. We show dependence between LE and coefficient of structural properties system (CSP). Then we described modes of identification SE on the bas is of the estimations LE. We considered type of roots linear dynamic system. Further we offered a method of an estimation LE for non-stationary system on the basis of the analysis CSP. We described a mode of estimation the largest LE. $S_{\mathcal{L E}}$ - structure. The method is based on the analysis of properties offered in work $S_{\mathcal{L E}}$-structure.

\section{PROBLEM ST ATEMENT}

Consider linear dynamic system

$$
\begin{aligned}
& \dot{X}=A X+B U, \\
& Y=C X+D U,
\end{aligned}
$$

where $X \in R^{m}$ is a state vector, $U \in R^{k}, Y \in R^{n}$ are an input and an exit of system, $A \in R^{m \times m}, B \in R^{m \times k}$,
$C \in R^{n \times m}, D \in R^{n \times k}$.

For (1) we have the experimental information

$$
\mathrm{I}_{o}=\left\{Y(t), U(t), t \in J=\left[t_{0}, t_{1}\right]\right\} .
$$

Note a solution of system (1)

$$
X(t)=X\left(t_{0}, U, t\right),
$$

where $X$ is an operator definitely defined by matrixes $A, B$.

On the basis of (2) from (3) we receive a solution of $\operatorname{system(1)}$ at $X_{0}=X\left(t_{0}\right)$

$$
X(t)=X_{g}(t)+X_{q}(t)
$$

where $X_{q}(t)$ a particular solution (1) with $U \in \mathrm{I}_{o}$, $X_{g}(t)$ is a general solution (1) with $U(t)=0$ at the unknown $X_{0} \in \mathrm{I}_{o}$. Designate $X_{g}\left(X_{0}, t\right)$ as the general solution (1) with $X_{0}=X_{0}\left(Y_{0}\right) \in \mathrm{I}_{o}$.

The problem consists in determination of estimation a solution $X_{g}(t)=X_{g}\left(X_{q}, X_{0}, t\right)$ on set $\mathrm{I}_{o}$ and decisionmaking on an equilibrium state.

\section{IDENTIFICATION OF EQUILIBRIUM STATE (1)}

\section{A. Approach to problem solution}

We will divide procedure of a solution the task into two stages: (i) identification of a solution $X_{q}(t)$, (ii) determination $X_{g}(t)$ as functions from $X_{q}(t)$. Selection of a particular solution on a class of dynamic systems (1) is complex task. Therefore we fulfilled identification on a class of static models for finding $X_{q}(t)$. We searched dependence $X_{q}(t)=X_{q}(U, t)$ on some interval $J_{q} \subset J$. We can apply various approaches to a solution of this problem. The model choice depends on a frequency spectrum $Y(t)$.

We will explain a method of solution a problem for system (1) second order [21]. Consider system (1) with one input and an output. Designate $y=Y, u=U, y \in R$, $u \in R$.Let $D_{y}(\omega), D_{u}(\omega)$ are frequency spectrums $u, y$. We suppose $\|y(t)\|<\infty,\|u(t)\|<\infty$. Let $D_{y}(\omega)=D_{u}(\omega)$. Hence the system (1) is linear and stationary. Let roots $\lambda_{i}$ of a characteristic equation system (1) were negative $\lambda_{i} \leq 0 \quad \forall i=1,2$.

Write $\mathrm{I}_{o}$ as

$$
\mathrm{I}_{o}=\mathrm{I}_{o}^{q}\left(J_{q}\right) \bigcup \mathrm{I}_{o}^{g}\left(J_{g}\right),
$$

where $J_{q} \cup J_{g}=J \subseteq R ; \mathrm{I}_{o}^{q}, \mathrm{I}_{o}^{g}$ are the sets containing the information about $X_{q}$ and $X_{g}$.

On set $\mathrm{I}_{o}^{q}\left(J_{q}\right)$ we estimate the separate particular solution of system (1). As $x_{1}=y \in R$, to deriving of a com 
ponent $x_{2}=\dot{x}_{1}$ of a vector $X$ apply operation of differentiation a variable $y$. We will designate $\hat{x}_{2}=\dot{y}$. Apply static model to an estimation of a particular solution system (1)

$$
\hat{X}_{q}(t)=\hat{V} W(t),
$$

where $\hat{V} \in R^{2 \times 2}$ is a matrix of parameters of model, $W=\left[\begin{array}{ll}u & u^{\prime}\end{array}\right]^{T}$.

We will use the least-squares method for determination $\hat{V}$. Knowing $\hat{X}_{q}$, define $\hat{X}_{g}$

$$
\hat{X}_{g}=\hat{X}_{q}-\hat{X}
$$

where $\hat{X}=\left[\begin{array}{ll}y & \hat{x}_{2}\end{array}\right]^{T}, \hat{X}_{g}=\left[\begin{array}{ll}\hat{y}_{g} & \hat{y}_{g}^{\prime}\end{array}\right]^{T}=\left[\begin{array}{ll}\hat{x}_{g, 1} & \hat{x}_{g, 2}\end{array}\right]^{T}$.

After receive of a vector $\hat{X}_{g}$ we will construct map $\Gamma_{g}:\left\{\hat{y}_{g}\right\} \rightarrow\left\{\dot{\hat{y}}_{g}\right\}$ to a phase plane and we define type of a point an equilibrium state.

We will apply the following approach to determination of an estimation type of a critical point in system (1). Define a partial derivative

$$
F_{g}=\frac{\partial X}{\partial u}=\left[\frac{\partial y}{\partial u} \frac{\partial \hat{x}_{2}}{\partial u}\right]^{T}=\left[f_{1} f_{2}\right]^{T} .
$$

Construct map $\Gamma_{g}^{t}:\left\{f_{1}\right\} \rightarrow\left\{f_{2}\right\}$ which name $F$-characteristic of system (1). For linear system (1) we will write vector $F_{g}$ as

$$
F_{g}=\int_{t_{0}}^{t} e^{A(t-\tau)} B d \tau
$$

$F_{g}$ is a function of the exponents dependent on eigenvalues of system, and also secular terms and sinusoid. (6) we will name $F$-system.

Definition. The behavior of two dynamic systems qualitatively coincides on a phase plane if they have the same type of an equilibrium state.

Statement 1. For system (1) map $\Gamma_{g}^{t}$ qualitatively coincides with $\Gamma_{g}$.

Proof. The equation (6) described dynamic system. Therefore $F_{g}$ is a vector of a state system (1) with $u(t)=1$ and zero initial conditions. Systems (1) and (6) have an identical spectrum of eigenvalues. The statement is proved.

Distinction between systems (1) and (6).

1. For system (1) entry conditions are unknown, and for system (6) they are zero. This difference is reflected in the initial stage of a modification maps $\Gamma_{g}^{t}$ and $\Gamma_{g}$.

2. For $F$-system we receive an estimation of the general solution with application described above the approach.

3. Representation of map $\Gamma_{g}^{t}$ depends on a choice of interval $J$.

So, we have offered two approaches to an estimation of type an equilibrium state of dynamic system. The first approach is based on identifications of a particular solution system on a class of static models. The second approach is based on determination $F$ - characteristics of system.

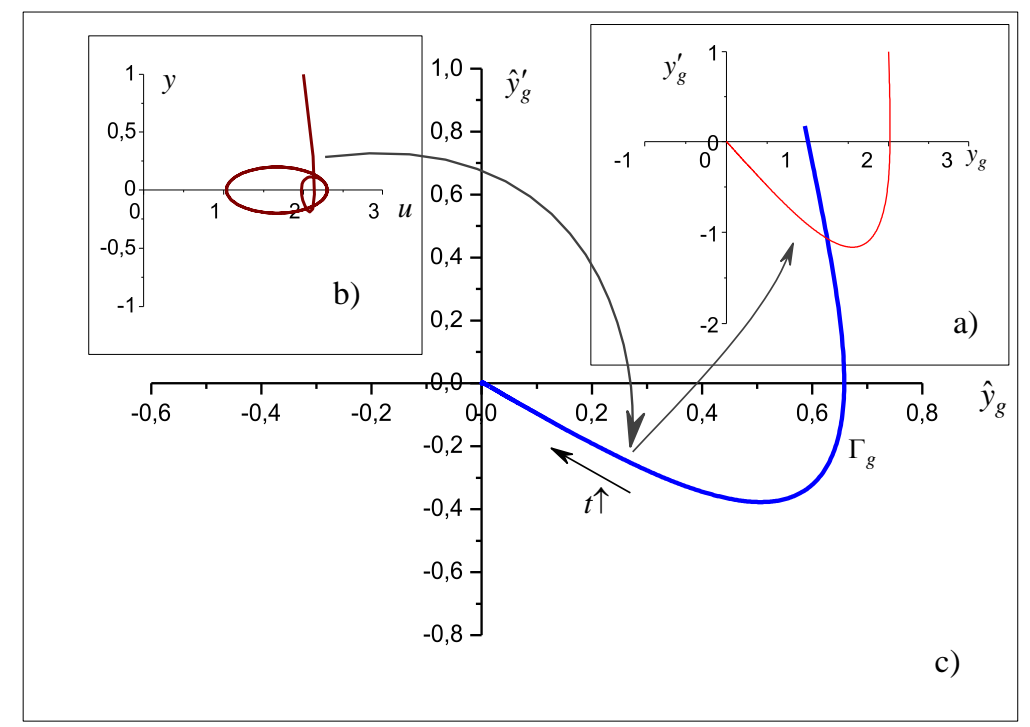

Fig. 1. Estimation of an equilibrium state for linear stationary dynamic system of the second order with $\lambda_{i}<0$

\section{B. Examples of identification equilibrium state}

Consider linear system (1) second order with a sinusoidal input. Fig. 1 showed results of identification type a critical point of system with $\lambda_{1}<0, \lambda_{2}<0$. Fig. 1a shows a response of system (1) on entry conditions at $u(t)=0$. The Fig. 1b shows an observable informational 
portrait (OIP) [11] systems (1) on a plane $(u, y)$. On Fig. 1c we have presented an estimation of free movement system (phase portrait) (1). From a figure we see that the system has a stable node. Fig. 2 showed $F$ - characteristic of system(6).

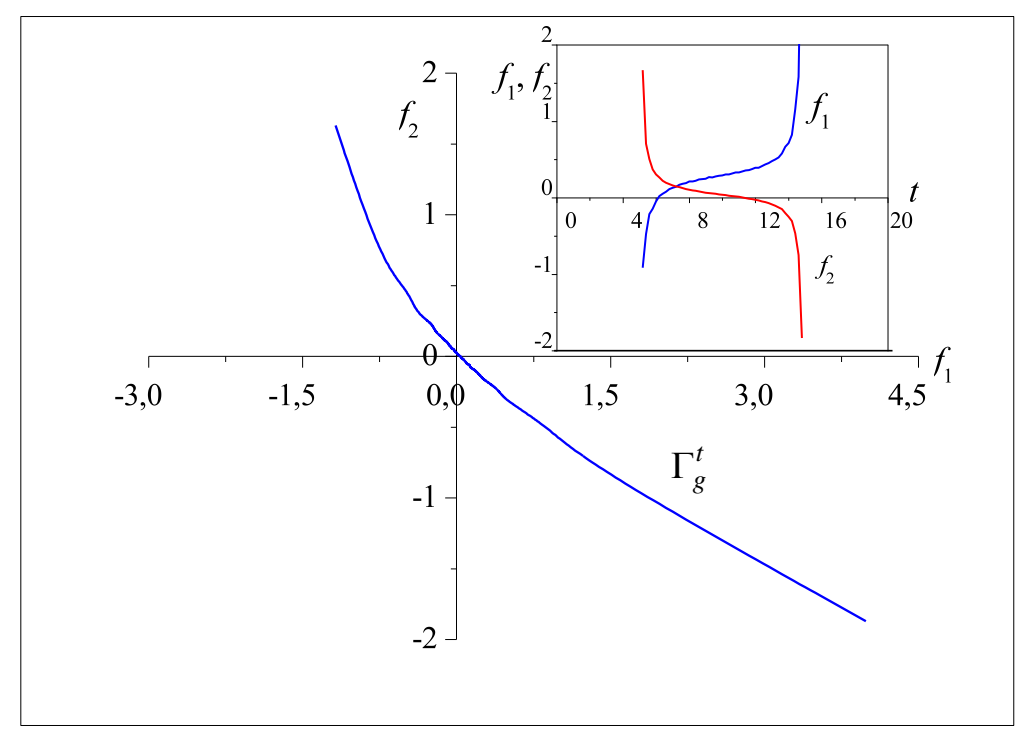

Fig. 2. $F$-characteristic of linear dynamic system the second order with $\lambda_{i}<0$

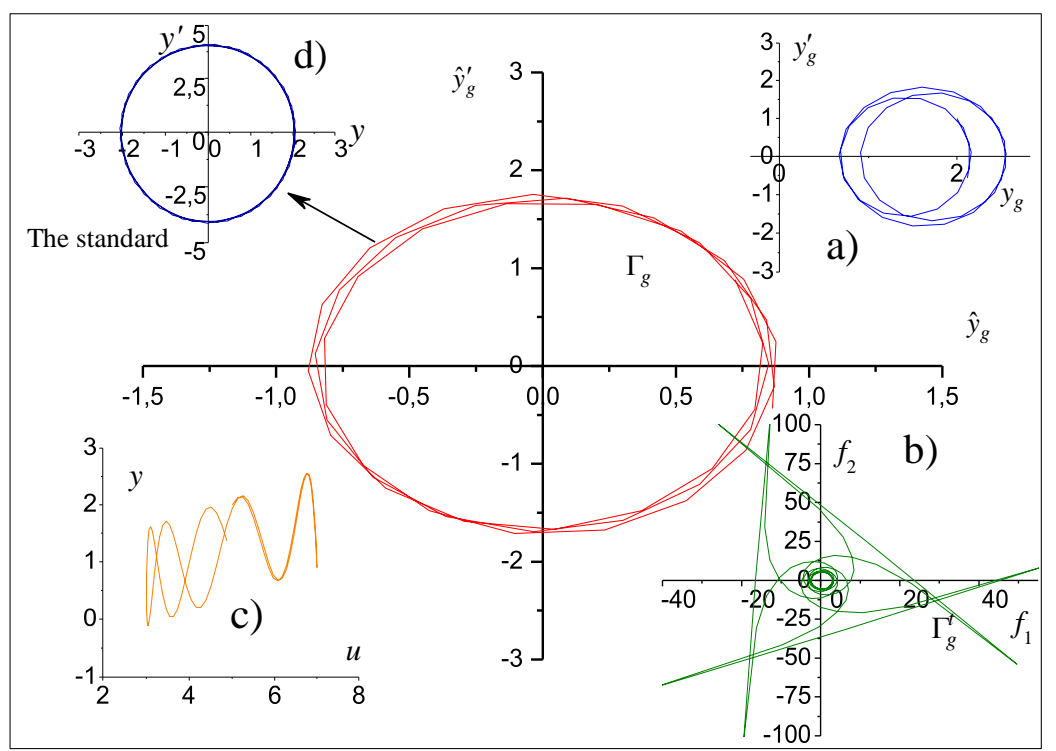

Fig. 3. The equilibrium state of linear stationary dynamic system of the second order with imaginary roots

Fig. 3 represented results of identification system of the second order with imaginary roots. On Fig. 3a we show the phase portrait of system received on the basis of the analysis set $\mathrm{I}_{o}$. On fig. $3 \mathrm{c}$ we show OIP. The Fig. $3 \mathrm{~b}$ showed $F$-characteristic the system. On fig. $3 d$ the reference phase portrait of system is shown. From a figure we see that both modes state the same estimation of an equilibrium state. It is the centre.

On Fig. 4 we represent results of estimation a point of an equilibrium state system of the second order with complex roots with the real negative parts. The equilibrium state is a stable focus.
So, results of modelling have confirmed working capacity of the offered approach.

\section{Criteria of estimation type equilibrium state}

We will describe criteria of an estimation a point of an equilibrium state on the basis of results identification as they does not demand knowledge of map $\Gamma_{g}$.

Let we have informational set $\mathrm{I}_{\hat{X}_{g}}=\left\{\hat{X}_{g}(t), t \in J\right\}$. It is necessary to make a solution on an equilibrium state on the basis of the analysis $\mathrm{I}_{\hat{X}_{g}}$. 


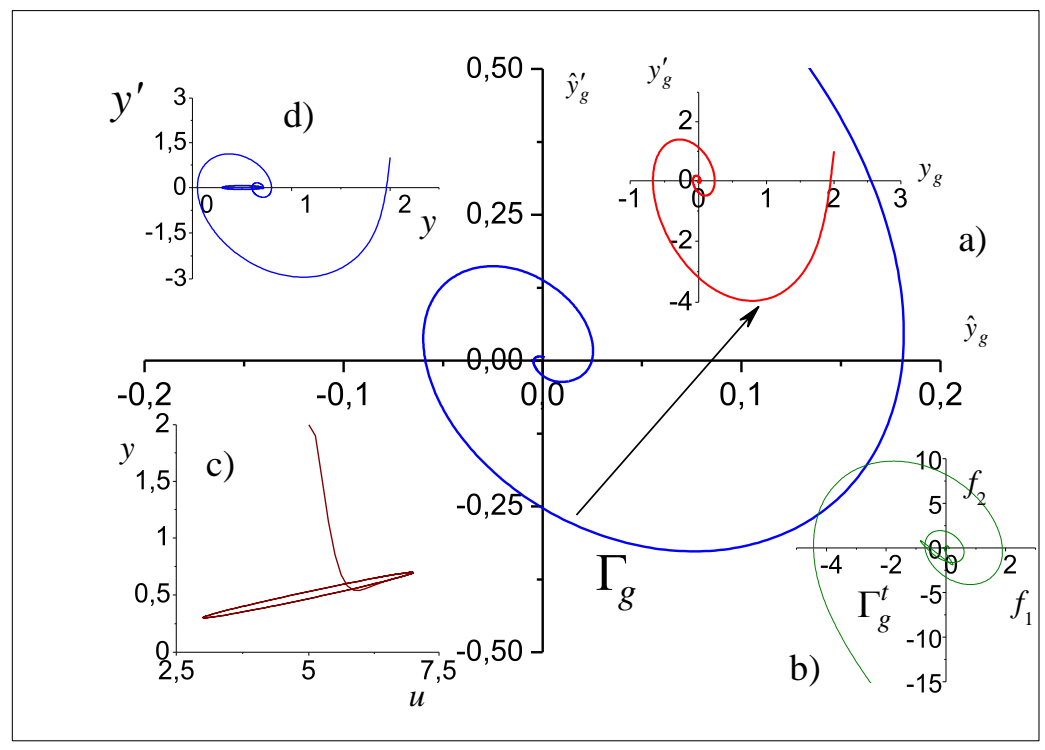

Fig. 4. The equilibrium state of linear stationary dynamic system of the second order with complex roots

For a curve, described by the equation $f(v, z)=0$, conditions of existence a critical point $M_{0}\left(v_{0}, z_{0}\right)$ write as [26]

$$
\begin{aligned}
& f\left(v_{0}, z_{0}\right)=0, \\
& \frac{\partial}{\partial v} f(v, z)=0, \frac{\partial}{\partial z} f(v, z)=0 .
\end{aligned}
$$

These was sufficient conditions. They allow defining only an equilibrium state for the elementary dynamic systems. The node is an example of such equilibrium point. We described curve (map $\Gamma_{g}$ ) on a phase plane in our case in the parametric form $\hat{X}_{g}=\Psi(t)$. Therefore for system of the second order we will write a condition of existence of a critical point $M_{0}\left(\hat{x}_{g, 1}^{0}, \hat{x}_{g, 2}^{0}\right)$ as

$$
\Psi\left(t^{0}\right)=0, \Psi^{\prime}\left(t^{0}\right)=0
$$

where $\hat{x}_{g, 1}^{0}=x_{g, 1}\left(t_{*}\right), \hat{x}_{g, 2}^{0}=x_{g, 2}\left(t_{*}\right) ; t_{*}>t_{0}$ is an instant when conditions (8) was correctly.

1. Criterion of definition a stable node. If the vector $\hat{X}_{g}(t)$ is smooth function for almost all $t \in J$, and its derivative $\hat{X}_{g}^{\prime}(t)$ continuously decreases and for some $t_{*} \in J$

$$
\hat{X}_{g}^{\prime}\left(t_{*}\right)=0, \hat{X}_{g}\left(t_{*}\right)=0,
$$

then the system (1) has an equilibrium state a stable node.

The first condition (9) relaxes condition (8) as operation of numerical differentiation is very sensitive to errors. The second condition (9) directly follows from critical point definition.

Remark 1. As we searched a solution on set $\mathrm{I}_{\hat{X}_{g}}$, we identified a point $M_{0}$ with orig in of coordinates on a phase plane.

2. Criterion of definition a stable focus. If the vector $\hat{X}_{g}(t)$ is smooth function for almost all $t \in J$, and its derivative $\hat{X}_{g}^{\prime}(t)$ continuously decreases and at sufficiently great $t_{*}>t_{0} \in J$

$$
\hat{X}_{g}^{\prime}\left(t_{*}\right) \rightarrow 0, \hat{X}_{g}\left(t_{*}\right) \in O_{M_{0}},
$$

where $O_{M_{0}}$ is a neighbourhood of a point $M_{0}$, then the system(1) has an equilibrium state a stable focus.

In this case conditions (9) are not fulfilled. As the vector $\hat{X}_{g}^{\prime}(t)$ decreased, it reached a point 0 only at sufficiently great values $t$. If map $\hat{X}_{g}\left(t_{*}\right) \subset O_{M_{0}}$ correctly, the critical point are stable focus.

3. Criterion of definition the centre. If vectors $\hat{X}_{g}(t)$, $\hat{X}_{g}^{\prime}(t)$ are smooth functions for almost all $t \in J_{g}$ and

$$
\left\|\hat{X}_{g}(t)\right\| \geq \alpha_{g}, \quad\left\|\hat{X}_{g}^{\prime}(t)\right\| \geq \beta_{g} \quad \forall t \in J_{g},
$$

where $\alpha_{g}>0, \beta_{g}>0,\|\cdot\|$ is a norm of a vector, then the system(1) has an equilibrium state the centre.

D. About estimation of equilibrium state of linear nonstationary system

Consider system (1) with a piecewise continuous matrix $A=A(t),\|A(t)\|<\infty$. Let the matrix $A(t)$ is $\mathcal{T}$ hurwits. Then for $\forall t \geq t_{0}$ the condition is satisfied $\operatorname{Re}\left(\lambda_{i}(t)\right) \leq 0$, where $\lambda_{i}(t) \in \sigma(A(t))$ is eigenvalue $A(t), \sigma(A(t))$ is SE.

We will apply the method described above to identification of the general solution (1). Solution 
$X(t)=X\left(t_{0}, U, t\right)$ contains a non-stationary component which remains in $X(t)$ at large $t$. If the spectrum $\sigma(A(t))$ contains simple roots apply model (5) and receive an estimation of general solution $\hat{X}_{g} \cdot \hat{X}_{g}$ contained a nonstationarity, who will give to "smearing" of a point an equilibriu m state of a stable node. It gave oscillation of a trajectory about an equilibrium state.

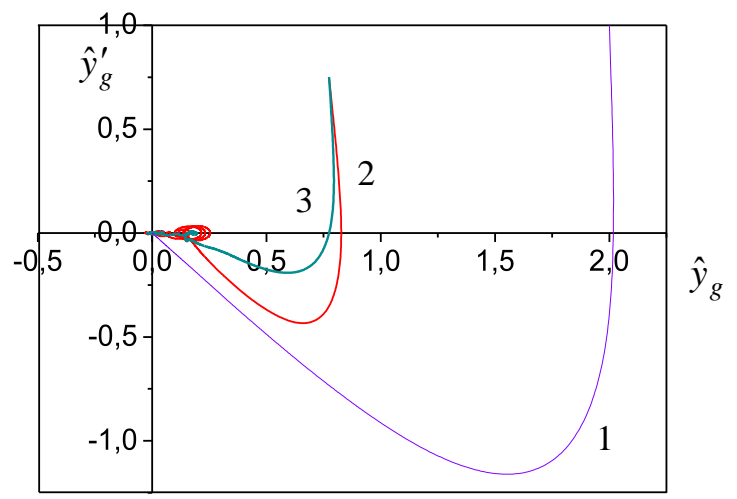

Fig. 5. Phase portrait of non-stationary system

Such phase portrait in the conditions of uncertainty is an indication of presence in system of a nonstationarity. It obviously we see at the analysis of $F$-system. In the conditions of uncertainty application of the offered approach gives only tentative estimations and we should use the additional information for decision-making. In particular we can apply LE. Further we state modes of identification Lyapunov exponents.

Fig. 5 showed a phase portrait (PP) for system (1) second order with a matrix

$$
A(t)=\left[\begin{array}{cc}
0 & 1 \\
-3+0.2 \sin (0.02 \pi x) & -4
\end{array}\right]
$$

and a sinusoidalinput.

We have seen that the nonstationarity gave smearing of equilibrium point of system. On Fig. 5 we have designated 1 as reference PP at $U(t)=0 ; 2$ as estimation PP received by means of model (5); 3 as the smoothed value of a curve 2. Application of procedure smoothing does not allow us to receive an ideal point of an equilibrium state system. The area of oscillation about an equilibrium state depended on a modification of parameter $a_{21}(t)$ a matrix A.

So, results of simu lation display an mismatch of conditions of an equilibrium for stationary and nonstationary systems.

Remark 2. We have carried out the analysis of scientific publications on the qualitative analysis of nonstationary dynamic systems. It has shown that the basic attention is given to research of a stability non-stationary system [1-5, 23-25]. Problems of construction PP had no development. Explain it complexity of dynamics the giv- en class of systems. Presence of uncertainty (nonstationarity) es sentially complicates the given problem. In this case as the additional information we can use the analysis of a spectrum eigenvalues of system.

\section{ESTIMATION OF SPECTRUM $\sigma(A)$ SYSTEM (1)}

We will apply Lyapunov exponents [27] to estimation SE of dynamic system (1). LE are a stability criterion of systemand for a real-valued function $h(t)$ are defined as

$$
\chi[h]=\varlimsup_{t \rightarrow \infty} \frac{\ln |h(t)|}{t},
$$

where $\varlimsup_{t \rightarrow \infty}$ is a superior limit.

$$
\chi_{i}(i=\overline{1, m}) \text { a nontrivial solution of system (1) coin- }
$$

cides with real parts of eigenvalues $\lambda_{i}$ of a matrix $A$. We will apply LE to definition of type an equilibrium point of system (1). Let the estimation of the general solution $X_{g}(t) \forall t \in J_{g}$ of system (1) is known. We assume that the system is stable. Apply (11) to $\hat{y}_{g}(t)$ and receive

$$
\chi\left[\hat{y}_{g}\right]=\varlimsup_{t \rightarrow \bar{t}} \frac{\ln \left|\hat{y}_{g}(t)\right|}{t},
$$

where $\bar{t} \in J_{g}$ is a upper boundary on an interval $J_{g} \subset J$.

If the limit (11) existed, $\chi\left[\hat{y}_{g}\right]$ is an estimation of the largest eigenvalue of a matrix $A$. Hence, $\chi\left[\hat{y}_{g}\right]$ is an estimation of degree of a stability system (1). Hence, $\chi\left[\hat{y}_{g}\right]$ is an estimation of a degree stability system(1). If $m=2$, then for $\dot{\hat{y}}_{g}$ receive

$$
\chi\left[\dot{\hat{y}}_{g}\right]=\varlimsup_{t \rightarrow \bar{t}} \frac{\ln \left|\dot{\hat{y}}_{g}\right|}{t} .
$$

On a basis $\chi\left[\hat{y}_{g}\right], \chi\left[\dot{\hat{y}}_{g}\right]$ we make a solution on type of an equilibrium point of system. We could use methods of identification for a raise of a calculation accuracy of LE.

The idea of application Lyapunov exponents in identification problems is stated in [21, 22]. The offer method of definition LE is based on the analysis of coefficient structural properties [11]. We give development of the given method. We will show at first relationship between CSP and LE.

\section{A. About interdependence LE and CSP}

Calculate for system(1) indicator

$$
\rho\left(\hat{y}_{g}\right)=\ln \left|\hat{y}_{g}(t)\right| \quad \forall t \in \bar{J}_{g} \subset J_{g},
$$

where $\bar{J}_{g}=\left[t_{0}, \bar{t}\right]$ define on the basis of (12). 
Consider now system with an input $t$ and an output $\rho\left(\hat{y}_{g}\right)$. For an estimation of structural properties of this systemintroduce a coefficient of structural properties

$$
k_{s}(t, \rho)=\frac{\rho\left(\hat{y}_{g}\right)}{t} \text {. }
$$

$k_{s}(t, \rho)$ is a basis for a calculation of an indicator $\chi\left[\hat{y}_{g}\right](12)$ on an interval $\bar{J}_{g}$.

We consider $k_{s}(t, \rho)$ as a transmission factor of system estimation LE with an input $t$ and an output $\rho\left(\hat{y}_{g}\right)$. Further we will show as $k_{s}(t, \rho)$ to apply to an estimation largest LE.

So, we have defined interdependence between the Lyapunov exponent $\chi\left[\hat{y}_{g}\right]$ and a coefficient of structural properties $k_{s}(t, \rho)$ on informational set $\mathrm{I}_{\rho}=\left\{\rho\left(\hat{y}_{g}(t)\right), t \in \bar{J}_{g}\right\}$.

Consider set

$$
\mathrm{I}_{g}\left(\hat{y}_{g}, t\right)=\left\{\hat{y}_{g}(t), t \in J_{g}\right\}=\mathrm{I}_{\hat{x}_{g}} \backslash\left\{\dot{\hat{y}}_{g}(t), t \in J_{g}\right\},
$$

containing the data about a variable modification $\hat{y}_{g}$ on an interval $J_{g}$.

We suppose that the system (1) is stable, $\operatorname{Re}\left(\lambda_{i}\right) \leq 0$, $i=\overline{1, m}$, where $\lambda_{i} \in \sigma(A)$.

Problem: on the basis of the analysis of sets $\mathrm{I}_{\rho}, \mathrm{I}_{g}$ estimate a spectrum $\sigma(A)$ of eigenvalues a matrix $A$ of system(1).

Problem solution reduces to realization of following stages.

1. Definition of dimension a spectrum $\sigma(A)$.

2. Deriving of initial estimations for a matrix spectrum A.

3. Design of the adaptive algorithms for an improvement of the estimations received at a stage 2 .

Adaptive algorithm was described in [29]. Therefore we had done not consider them.

The main difficulty of application of the least-squares method is approximation $\hat{y}_{g}(t)$ on a class of functions $\left\{e^{\lambda_{i} t}, i=\overline{1, n}\right\}$ which nonlinearly depended from $\lambda_{i}$.

Consider each of subtasks. The solution of a problem 1 can be received by means of the theorem 3.1 [21] on the basis of the set analysis $\mathrm{I}_{o}$.

At a solution of a problem 2 we consider type of roots dynamic system. We will consider some cases.

\section{B. Simple eigenvalues}

Let matrix eigenvalues $A$ are arranged in decreasing order $\lambda_{1}>\lambda_{2}>\ldots>\lambda_{m}$. On the basis of (12), (13) define an estimation of a largest eigenvalue $\lambda_{1}$ of a matrix $A$

$$
\chi\left[\hat{y}_{g}\right]=\lambda_{1}=\varlimsup_{t \rightarrow \bar{t}} \frac{\ln \left|\hat{y}_{g}(t)\right|}{t},
$$

$\rho(t)=\rho\left(\hat{y}_{g}\right)$ could have the irregular character because of calculation errors. Therefore apply procedure of an average $\rho(t)$ on some interval $\bar{J} \subset J_{g}$ to determination $\lambda_{1}$. Other approach to an estimation $\lambda_{1}$ reduce to following operations.

Consider map $\Gamma_{\rho}: t \rightarrow \rho$ to planes $(t, \rho)$ and define a secant $\bar{\gamma}_{\rho}[11]$

$$
\bar{\gamma}_{\rho}=\bar{\gamma}(t, \rho)=a_{0}^{\rho}+a_{1}^{\rho} t
$$

where numbers $a_{0}^{\rho}, a_{1}^{\rho}$ define by means of method least-squares.

Statement 2 [21, 22]. $\lambda_{1}=a_{1}^{\rho}$.

As $\hat{y}_{g}(t)$ is the sum of exponents, sinusoids or seculars terms, then it is true [27] $\chi\left[\hat{y}_{g}\right] \leq \lambda_{1}$. Therefore for definition of estimations $\lambda_{i}, i=\overline{2, m}$ we make the additional analysis of function $\chi\left[\hat{y}_{g}(t)\right]$.

Write $\hat{y}_{g}(t)$ as

$$
\hat{y}_{g}(t)=c_{1} e^{\lambda_{1} t}+c_{2} e^{\lambda_{2} t}+\zeta_{3}=c_{1} e^{\lambda_{1} t}\left(1+c_{12} e^{\Delta \lambda_{12} t}+v_{3}\right),
$$

where $v_{3}=\zeta_{3} e^{-\lambda_{1} t} / c_{1}, \zeta_{3}$ is a sum of exponent with $\lambda_{i}(i \geq 3), \Delta \lambda_{12}=\lambda_{2}-\lambda_{1}, c_{12}=c_{2} / c_{1}$.

Consider the sum $1+c_{12} e^{\Delta \lambda_{12} t}$. We neglect a summand $v_{3}$ as it decreases faster $e^{\Delta \lambda_{12} t}$. Use an inequality $a^{2}+b^{2} \geq 2 a b$ and (15) write as

$$
\hat{y}_{g}(t) \geq 2 c_{1} e^{\lambda_{1} t} d_{12} e^{0.5 \Delta \lambda_{12} t},
$$

where $d_{12}=\sqrt{c_{12}}, \Delta \lambda_{12}=\lambda_{2}-\lambda_{1}$. Then

$$
\rho(t) \cong \ln \left(2 c_{1}\right)+\lambda_{1} t+\ln \left(d_{12}\right)+0,5 \Delta \lambda_{12} t \text {. }
$$

Divide (16) on $t$. Then on an interval $J_{2} \subset J$ receive

$$
\delta_{2}=\left.S_{2}(\rho(t))\right|_{t \in J_{2}}-\lambda_{1}=0,5 \Delta \lambda_{12},
$$

where $S_{2}(\rho(t))$ is the average value $\rho(t)$ received by means of an operator $S_{2}$ on an interval $J_{2}$.

From (17) receive

$$
\lambda_{2}=2 \delta_{2}+\lambda_{1} \text {. }
$$

As we search initial approximations for $\lambda_{i}(i \geq 2)$ the formula (18) can use for finding of other elements of a spectrum of a matrix $A$. We analyze CSP [21] to choose an interval $J_{i}$ and values $\delta_{i}$.

Consider function $v(t)=\rho(t) / t$, construct a secant

$$
\bar{\gamma}(t, v)=a_{o}^{v}+a_{1}^{v} t
$$


and introduce error function $\delta(t)=v(t)-\bar{\gamma}(t, v)$.

Analyze a modification $\delta(t)$ and find intervals $J_{i} \subset J$ on which function $\delta(t)$ has local minimums. We accept these values for magnitudes $\delta_{i}$. Apply operation of an average $S_{i}(v(t))$ to decrease of computing errors $\delta(t)$. Calculate

$$
\lambda_{i}=2 \delta_{i}+\lambda_{1}
$$

and define an estimation $i$-th eigenvalue of a matrix A.

For decision-making on efficiency of the received estimations introduce function $\pi_{i}(t)=e^{\lambda_{i} t}$ and calculate a coefficient of determination $r_{\hat{y}_{g} \pi_{i}}^{2}$. If

$$
r_{\hat{y}_{g} \pi_{i}}^{2} \geq \kappa
$$

where $\kappa>0$ is some specify magnitude, we accepted estimation $\lambda_{i}$ as the initial. Otherwise consider other interval $J_{i}$ and apply (19) or fulfil value correction $\lambda_{i}$, that it was true (20).

If function $\delta(t)$ is monotone to for determination $\lambda_{i}$ apply to $\dot{\hat{y}}_{g}(t)$ described a method. If function $\delta(t)$ are the monotonic, then the described method apply to $\dot{\hat{y}}_{g}(t)$ finding $\lambda_{i}$. We will describe further criteria of decisionmaking on eigenvalue type.

So, we offer the approach to finding of initial estimations for a spectrum $\sigma(A)$ of a matrix $A$ in case of simple roots.

Remark 3. The problem of an estimation the largest LE depended on a choice of value $\bar{t}$ in (14). We offer a method of solution this problem on the basis of the analysis a secant $\bar{\gamma}(t, v)$. In section 5 we introduce the special structure $S_{\text {LE }}$ defined on set of CSP. The analysis $S_{\text {LE }}$ allows to find easier an estimation largest LE.

\section{Multiple eigenvalues}

The general solution system(1) writes in the form [29]

$$
\hat{y}_{g}(t)=\sum_{i=1}^{p} e^{\lambda_{i} t} P_{i}\left(t, s_{i}\right)
$$

where $\lambda_{i}$ is a root of a order $s_{i}, s_{1}+s_{2}+\ldots+s_{p}=m$, $P_{i}\left(t, s_{i}\right)$ is a polynomial of degree $s_{i}-1$.

We will state a method of estimation a spectrum $\sigma(A)$ on an example of one root $\lambda_{1}$ an order $s_{1}$ [21]. Write $\hat{y}_{g}(t)$ as

$$
\hat{y}_{g}(t)=e^{\lambda_{1} t}\left(c_{1}+c_{2} t+\ldots+c_{s_{1}} t^{s_{1}-1}\right) .
$$

Factor out $t^{s_{1}-1}$ and apply the approach stated above. Then a sufficiently large $t$ we will receive

$$
\chi\left[\hat{y}_{g}\right]=\lambda_{1}+\varlimsup_{t \rightarrow \bar{t}} \frac{\ln \left(t^{s_{1}-1}\right)}{t}=\lambda_{1}+\chi\left[t^{s_{1}-1}\right] .
$$

Calculate $\chi\left[t^{s_{1}-1}\right]$ and from (23) receive estimation

$$
\lambda_{1}=\chi\left[\hat{y}_{g}\right]-\chi\left[t^{s_{1}-1}\right] \text {. }
$$

So, we have offered the approach to an estimation of a spectrum in case of one multiple eigenvalue. In the presence of several multiple roots add the offered approach a method stated in section 4.B.

Consider a problem of determination multiplicity of a root $s_{1}$. We estimate dimension of system by means of the theorem 3.1 [21]. If the equations (21) or (22) are known, then definition of value $s_{1}$ does not represent computing problems. On the basis of the analysis sets $I_{g}$ or $\mathrm{I}_{\rho}$ we cannot offer any regular methods of an estimation $s_{1}$. On the basis of the analysis of sets $\mathrm{I}_{g}$ or $\mathrm{I}_{\rho}$ we could not offer the regular estimation methods $s_{1}$. Therefore we will state the approach based on the informational analysis of set $\mathrm{I}_{\rho}$.

Introduce function

$$
d(t)=\frac{\rho\left(\hat{y}_{g}\right)}{\rho\left(\dot{\hat{y}}_{g}\right)} .
$$

As $\hat{y}_{g}(t)$ is described by dependence (21) $d(t)$ in some points $t \in J_{g}$ will have singularities. Explain it to that $\hat{y}_{g}$ in these points has local extremums. It gives continuity violation $d(t)$. Further consider functions $e^{\lambda_{1} t} t^{i}$ $(i=1,2, \ldots)$ and choose such $i$ at which the maximum $e^{\lambda_{1} t} t^{i}$ coincides with a point of violation a continuity $d(t)$. Let $s_{1}=i$. Repeat the described procedure for other roots $\lambda_{i}$.

Remark 4. In case of simple eigenvalues function $d(t)$ is the monotone.

So, the basic indication of presence multiple roots in systemis loss of a monotony of function $d(t)$.

\section{Complex eigenvalues}

In this case in system we have periodic damped oscillations. Hence, function $\rho\left(\hat{y}_{g}\right)$ had a component which period of a modification will allow defining frequency of characteristic oscillations $\omega$. We will write an eigenvalue as $\lambda=-\alpha \mp j \omega$. Develop for finding of simple eigenvalues a method applies to an estimation $\alpha$.

Generalize the received results.

Statement 3. Let a set $I_{g}$ received as a result of handling experimental data $\mathrm{I}_{o}$, and function $d(t)$. Then:

i) if $d(t)$ a monotone function, then the system (1) had simple eigenvalues; 
ii) if function $d(t)$ contains a periodic component, then the system(1) had complex eigenvalues;

iii) if in some points $t \in J_{g}$ the condition of a monotonicity function $d(t)$ is not fulfilled, then the system (1) had multiple eigenvalues.

The statement 3 gives conditions for application of the offered methods.

For an improvement of the received estimations a spectrum eigenvalues of system (1) apply identification methods. Considering specificity of a considered problem, in [21] we have applied algorithms of adaptive identification to estimation $\sigma(A)$.

\section{E. Examples}

1. Consider linear system (1) second order with a sinusoidal input. A spectrum of eigenvalues system $\sigma(A)=(-1,-3)$. For system the informational set is known $\mathrm{I}_{o}$. We want to receive Lyapunov exponents and to estimate a spectrum of system on the basis of the set analys is $\mathrm{I}_{o}$.

On the basis of the analysis set $\mathrm{I}_{o}$ and the theorem 3.1 [21] we have defined an estimation of an order system: $m=2$. The set $\mathrm{I}_{g}$ on an interval $J_{p}=[0 ; 7.8] \mathrm{sec}$ is generated and functions $v(t)$ and $\rho(t)$ (Fig. 6) are defined.

From Fig. 6 we see that $\rho(t)$ is a monotonic function. Therefore we have applied the approach offered in section 4.B to the analysis of functions $\rho_{d}(t)=\ln \left|\dot{\hat{y}}_{g}(t)\right|$, $v_{d}(t)=\rho_{d}(t) / t$. The analysis of a modification of function $d(t)$ has shown that it has monotone character. Therefore the considered systemhas simple roots.

We have constructed a secant $\bar{\gamma}_{\rho_{d}}$ and have defined an estimation of the largest eigenvalue $\hat{\lambda}_{1}=a_{1}^{\rho_{d}}=-1.04$. The estimation $\lambda_{1}$ received on the basis of an average $\rho(t)$, and it is -0.85 . For estimation finding $\lambda_{2}$ choose an interval $J_{2}$ and magnitude $\delta_{2}$ in (17). Analyze a modification of function $\rho(t)$ on Fig. 6. On the basis of the analysis we have selected two intervals $J_{2}^{1}, J_{2}^{2}$. The interval $J_{2}^{2}$ is located in the end $J_{g}$. We have done not analyze an interval $J_{2}^{2}$ so on it we define an estimation $\lambda_{1}$. On $J_{2}^{1}=[0.4 ; 1] \mathrm{sec}$ we have defined magnitude $\delta_{2}^{1}=-0.9$ and on it by means of (18) have received an estimation $\hat{\lambda}_{2}=-2.8$.

For definition of efficiency an estimation of a spectrum $\sigma(A)$ construct model

$$
\overline{\hat{y}}_{g}(t)=\beta_{1} \pi_{1}(t)+\beta_{2} \pi_{2}(t)
$$

with variables $\pi_{i}=\exp \left(\hat{\lambda}_{i} t\right), i=1,2$. Calculate a coefficient of determination between variables $\overline{\hat{y}}_{g}$ and $\hat{y}_{g}$. It is equal 0.95 .

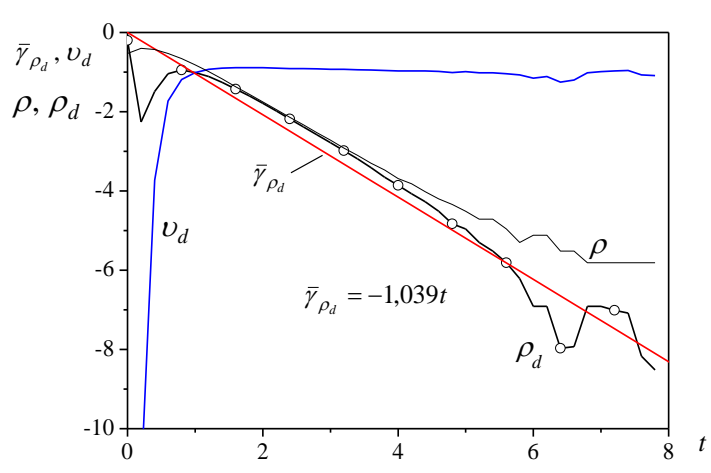

Fig. 6. Functions $v(t), \rho(t)$

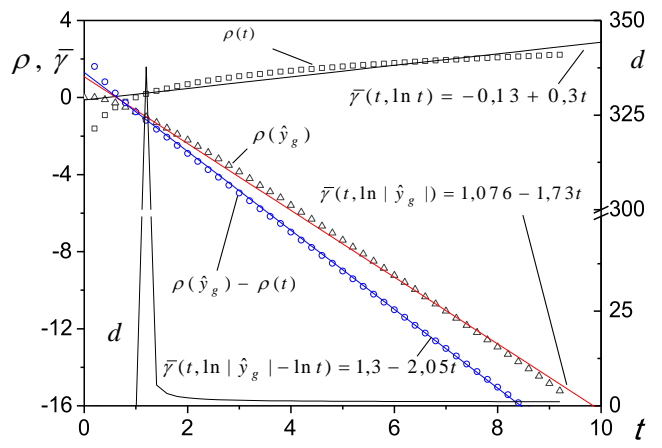

Fig. 7. Estimation of spectrum system with multiple roots

2. Consider dynamic system (1) second order with multiple roots $\lambda_{1}=\lambda_{2}=-2$. The Fig. 7 will show function $d(t)$. From Fig. 7 we see that $d(t)$ on some interval there is a nonmonotonic function. The estimation $\lambda$ received by means of (23), is equal -1.9. Application of functions $\rho\left(\hat{y}_{g}\right), \quad \rho(t)$ and secants gives $\lambda_{1}=$ $\lambda_{2}=-2.05$ (Fig. 7).

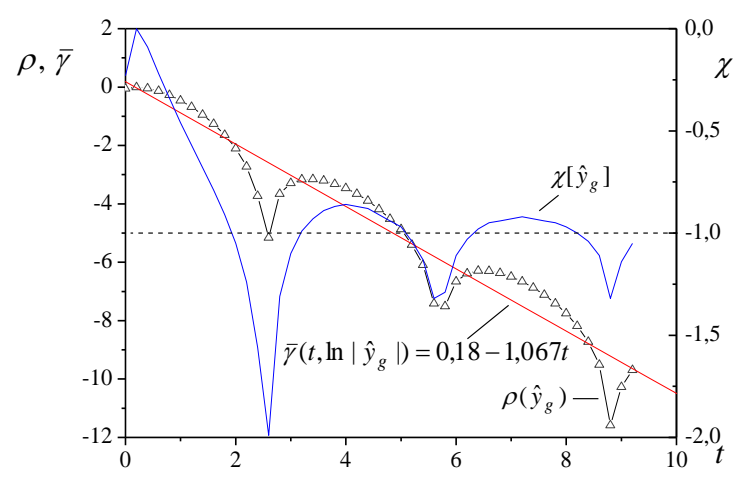

Fig. 8. Results of identification system with complex roots

3. Consider system of the second order with the matrix of a state having a spectrum $\sigma(A)=(-1+j,-1-j)$. Results of an estimation a real part eigenvalues show on Fig. 8. Application of secants and (14) is given practically identical values for $\operatorname{Re}(\lambda)$. For definition of frequency a 
modification oscillations in system on a basis Fig. 8 we have defined their period.

So, results of modelling confirm working capacity of the offered methods an estimation a spectrum of linear dynamic system.

We have stated only ideas of the approach which demand the further development and generalization. At identification of roots at first we should identify type of a point an equilibrium state.

\section{ESTIMATION LE FOR NONST ATIONARY SYSTEM}

Consider systems (1) with $A=A(t)$. We have described restrictions on a matrix $A(t)$ section 3.D. The limit superior choice in (14) is a complicated problem. We will apply the following method to estimation LLE.

Consider informational set

$$
\mathrm{I}_{\mathcal{L E E}}=\left\{k_{s}\left(t, \rho\left(\hat{y}_{g}\right)\right), k_{s}\left(t, \rho\left(\dot{\hat{y}}_{g}\right)\right), t\right\},
$$

where $k_{s}\left(t, \rho\left(\hat{y}_{g}\right)\right)$ is a coefficient of structural properties (13). The set (24) contains the information on a modification $\chi\left[\hat{y}_{g}\right]$ at observance of some conditions.

Consider map

$$
\Gamma_{k_{\rho}}: k_{s}\left(t, \rho\left(\hat{y}_{g}\right)\right) \rightarrow k_{s}\left(t, \rho\left(\dot{\hat{y}}_{g}\right)\right)
$$

and structure $S_{\mathcal{L E}}$ corresponding to it. $\Gamma_{k_{\rho}}$ describes a trajectory of a modification the parameters reflecting current position $\chi\left[\hat{y}_{g}\right]$ and $\chi\left[\dot{\hat{y}}_{g}\right]$.

Ideally on some subset $\tilde{J} \subset J$ map $\Gamma_{k_{\rho}}$ should be linear (looked a segment $A B$ on Fig. 9).

As properties of dynamic system vary, on an interval $J_{\chi} \subset J \backslash \tilde{J}$ the trajectory $k_{s}\left(t, \rho\left(\dot{\hat{y}}_{g}\right)\right)$ gets to some limited area $\Omega_{\chi}$. Analyze a modification $k_{s}\left(t, \rho\left(\dot{\hat{y}}_{g}\right)\right)$ in $\Omega_{\chi}$ and on the basis of (14) make a solution about LLE of nonstationary system.

Consider the system of a second order stud in section 3.D. Fig. 9 show an example structures $S_{\mathcal{L E}}$ for this plant.

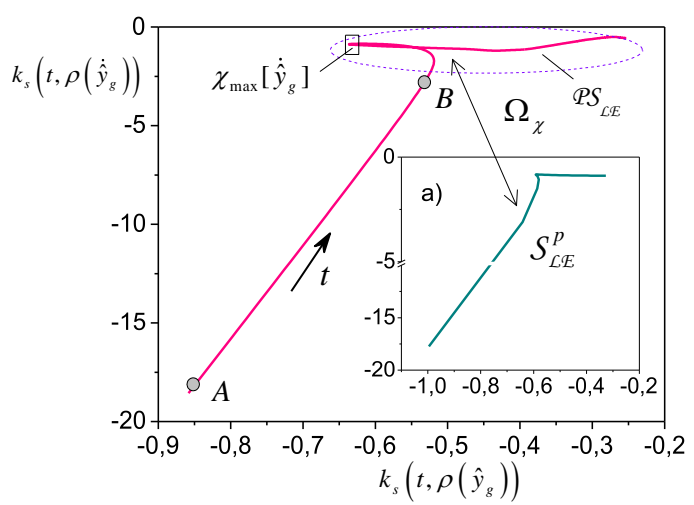

Fig. 9. $S_{L E}$-structure
Properties of structure $S_{\text {LE }}$.

1. The $S_{L \mathcal{E}}$-structure fragment $\mathcal{P} S_{\mathcal{L E}}$ contains the information about LLE.

2. The segment $A B$ contains the data for decisionmaking about $\chi_{i}\left[\dot{\hat{y}}_{g}\right], i \geq 2$.

3. The fragment $\mathcal{P S} S_{\mathcal{L E}}$ corresponds to area of an oscillation of a phase trajectory of system around equilibriu m position (Fig. 5).

To estimation $\chi_{i}\left[\dot{\hat{y}}_{g}\right]$ apply the approach stated in section 4.B. We will consider results of identification of Lyapunov exponents for an example reduced in section 3.D.

Estimations for Lyapunov exponents are $\chi_{1}\left[\dot{\hat{y}}_{g}\right]=-0.935, \chi_{2}\left[\dot{\hat{y}}_{g}\right]=-3.14$. For considered system $\lambda_{1}(t) \in[-1 ;-0.9], \lambda_{2}(t) \in[-3.1 ;-3]$.

Estimations for mean values $\lambda_{1}(t), \lambda_{2}(t)$ are

$$
\bar{\lambda}_{1}(t)=-0.931, \bar{\lambda}_{2}(t)=-3.07
$$

and coincide with $\chi_{i}\left[\dot{\hat{y}}_{g}\right]$.

For check of adequacy of the received estimations a spectrum construct model

$$
\hat{y}_{g, \chi}(t)=\alpha_{1} \exp \left(\chi_{1}\left[\dot{\hat{y}}_{g}\right] t\right)+\alpha_{2} \exp \left(\chi_{2}\left[\dot{\hat{y}}_{g}\right] t\right)
$$

for prediction $\hat{y}_{g}$, where $\alpha_{1}, \alpha_{2}$ define by means of a method of least squares. The coefficient of determination between $\hat{y}_{g}$ also $\overline{\hat{y}}_{g, \chi}$ is equal 0.99 .

For validation of correctness of application $S_{L E}$ for identification $\chi_{1}\left[\dot{\hat{y}}_{g}\right]$ we used models (25) and

$$
\dot{\hat{y}}_{g, \chi}(t)=\bar{\alpha}_{1} \exp \left(\chi_{1}\left[\dot{\hat{y}}_{g}\right] t\right)+\bar{\alpha}_{2} \exp \left(\chi_{2}\left[\dot{\hat{y}}_{g}\right] t\right) \text {. }
$$

On the basis of (25) and (26) we have generated set similar (24) with $k_{s}\left(t, \rho\left(\hat{y}_{g, \chi}\right)\right), k_{s}\left(t, \rho\left(\dot{\hat{y}}_{g, \chi}\right)\right)$ and have constructed structure $S_{\mathcal{L E}}^{p}$ (Fig. 9a). $S_{\mathcal{L E}}^{p}$ confirmed the validity of the offered approach.

So, we have offered the approach to identification LE for nonstationary dynamic systems. We have introduced structure $S_{\mathcal{L E}}$ on which basis have received an estimation of the largest Lyapunov exponent. To deriving of the least LE apply the results stated in section 4.

\section{CONCLUSION}

In work we have offered the approached methods of an estimation type a point of an equilibrium state linear $\mathrm{dy}$ namic system in the conditions of uncertainty. They are based on identification the particular solution of system on a class of static systems with the dynamic specification on a system input. We have applied these models and have received estimations of general solution (GS) systems. We have offered procedures and criteria of estima 
tion an equilibrium state on the basis of GS the system. These results are generalized on a case of linear nonstationary dynamic systems with simple eigenvalues.

At the second stage we solve a problem of estimation a spectrum eigenvalues of system on the basis of the time series analysis. Unlike existing approaches we introduce special space and the describe modification of Lyapunov exponents map. The method of the approached identification set Lyapunov exponents on the basis of the analys is this map is offered. Further we consider a case linear nonstationary dynamic systems c simple roots. The special structure for an estimation of the largest LE is introduced. We have shown that the structure has universal character and can be applied to the analysis of stationary linear dynamic systems.

\section{REFERENCES}

[1] D.K. Arrowsmith, C.M. Place. Ordinary differential equations. Chapman and Hall, London, New York, 1982.

[2] A. A. Andropov, E.A. Leontovich, I.I. Gordon, and A.G. Mayer. Qualitative theory of dynamic systems of second order. Nauka, Moscow, 1968.

[3] R. Reissig, G. Sansone, and R. Conti. Qualitative theorie nichtlinearer differentialgleichungen. Edizioni cremonese, Roma, 1974.

[4] L. Cesari. Asymptotic behavior and stability problems in ordinary differential equations. Springer -Verlag, Berlin, Gottingen, Heidelberg. 1959.

[5] N.N. Bautin, and E.A. Leontovich. Methods and modes of qualitative research of dy namic sy stems on a plane. Nauka, Moscow, 1990.

[6] L.N. Tikhonov, A.B. Vasil'eva, and A.G. Sveshnikov. Differential equations. Nauka, Moscow, 1980.

[7] P.G. Akishin, P. Akritas, I.Antoniou, and V.V. Ivanov. Identification of discrete chaotic maps with singular points. Discrete dynamics in nature and society, 2001, Vol. 6: 147156.

[8] J. Zhou, and F. Chen. A novel algorithm for detecting singular points from fingerprint images. IEEE Transactions on Pattern Analysis and Machine Intelligence, 2009, 31, 7: 1239-1250.

[9] D. Parekh, and R. Vig. Survey on parameters of fingerprint classification methods based on algorithmic flow. International Journal of Computer Science and Engineering Survey, 2011, 2, 3: 150-160.

[10] J.F. Foss, K.M. Bade, D.R. Neal, and R.J. Prevost. Topological considerations in support of PIV vector field analyses. 10th international symposium on particle image velocimetry - PIV13. Delft, Netherlands, July 1-3, 2013: 1-12.

[11] N.N. Karabutov. Adaptive identification of systems: Informational synthesis. URSS, Moscow, 2006.

[12] S. Ayasun, C.O. Nwankpa, and H.G. Kwatny. Computation of singular and singularity induced bifurcation points of differential-algebraic power system model. IEEE Transactions on Circuits and Sy stems I, 2004, 51, 8: 1525-1538.

[13] J. Meng, T. Zhu, X. Chen, and X. Yin. The nonlinear dynamics characteristics of stock market and its variation. Proceedings of the 2nd International Conference on Computer Science and Electronics Engineering (ICCSEE 2013). Published by Atlantis Press, Paris, France. 2013: 04500455.

[14] P.B. Kuptsov. Calculation of Lyapunov indexes for the distributed systems: advantages and shortages of various numerical methods. Informations of High Schools. Applied Nonlinear Dynamics. 2010,18, 5: 93-112.

[15] N.D. Poljahov, A.V. Bespalov, and V.E. Kuznetsov. Estimation of a condition of en gineering systems in real time on Lyapunov's first index. Conference materials «Control in engineering, erhatic, organizational network systems» (UTEOSS-2012). SCC the Russian Federation of Open Society "Concern TSNII "Electrodevice": Saint-Petersburg, 2012: 463-466.

[16] S. England. Quantifying dynamic stability of musculoskeletal systems using Lyapunov exponents. Blacksburg, Virginia. 2005.

[17] M.T. Rosenstein, J.J. Collins, C.J. De Luca. A practical method for calculating largest Lyapunov exponents from small data sets. Phy sica D. 1993, 65: 17-134.

[18] B.N. Datta, V.A. Yatsenko, and S.P. Nair. Model Updating and Simulation of Lyapunov Exponents. Proceedings of the European Control Conference 2007. Kos, Greece, July 2-5, 2007: 1094-1100.

[19] J.A. Scales, E.S. Van Vleck. Lyapunov exponents and localization in randomly layered media. journal of Computational Phy sics, 1997, 133: 27-42.

[20] K. Slevin, Y. Asada, L.I. and Deych. Fluctuations of the Lyapunov exponent in two-dimensional disordered systems. Phy sical Review B, 2004, 70, 054201: 054201-1054201-10.

[21] N.N. Karabutov. Structural Identification of Sy stems. The Analysis of Information Structures, URSS/ Librokom, Moscow, 2009.

[22] N.N. Karabutov. Deriving a dynamic-system eigenvalue spectrum under conditions of uncertainty by processing measurements. Measurement Techniques, Springer US, 2009, 52, 6: 572-579. DOI: 10.1007/ s11018-009-9309-0.

[23] L.E. Rejzin. Lyapunov's functions and a recognition problem. Zinatne, Riga, 1986.

[24] E.N. Rozenvasser Periodically non-stationary control systems. Nauka, Moscow, 1973.

[25] B.F. Bylov, R.E. Vinograd, D.M. Grobman, and V.V. Nemytsky. Theory of Lyapunov's indexes and its application to stability problems. Nauka, Moscow, 1966.

[26] P.K. Rashevsky. Course of differential geometry. State publishing house of the scientific and technical literature, Moscow, 1950.

[27] A.M. Lyapunov. General problem about movement stability. State publishing house of the scientific and technical literature, Moscow, 1950.

[28] N.N. Karabutov. Structural identification of systems: analy sis of dy namic structures. MSIU, Moscow, 2008.

[29] M.B. Fedorchuk. Ordinary differential equations. Nauka, Moscow, 1985.

\section{Author's Profiles}

Nikolay Karabutov is the professor of department Problem Control of Moscow state engineering university of radio engineering, Electronics and automation. The professor department of mathematics Financial University under the Government of the Russian Federation. Dr.Sci.Tech. The research areas are the automatic control theory, identification, adaptive control and decision-making.

How to cite this paper: Nikolay Karabutov,"Identification of Quality Indicators Dynamic System on Basis of Analysis Data "Input-Output"", IJISA, vol.6, no.9, pp.1-11, 2014. DOI: 10.5815/ijisa.2014.09.01 